\title{
Trends in Stroke Survival Incidence Rates in Older Australians in the New Millennium and Forecasts into the Future
}

\author{
Alexander Fisher, MD, PhD, Doc Med Sci, ${ }^{*}+$ Jodie Martin, BSc, $†$ \\ Wichat Srikusalanukul, MD, PhD, ${ }^{*}$ and Michael Davis, MBBS, FRACP ${ }^{*} \dagger$
}

\begin{abstract}
Aims: The objective of this study is (i) to evaluate trends in the incidence rates of stroke survivors aged 60 years and older over a 11-year period in the Australian Capital Territory (ACT) and (ii) to forecast future trends in Australia until 2051. Methods: Analysis of age- and sex-specific standardized incidence rates of older first-ever stroke survivors in ACT from 1999-2000 to 2009-2010 and projections of number of stroke survivors (NSS) in 2021 and 2051 using 2 models based only on (i) demographic changes and (ii) assuming changing of both incidence rates and demography. Results: In the ACT in the first decade of the 21st century, the absolute numbers and age-adjusted standardized incidence rates of stroke survivors (measured as a function of age and period) increased among both men and women aged 60 years or older. The trend toward increased survival rates in both sexes was driven mainly by population aging, whereas the effect of stroke year was more pronounced in men compared with women. The absolute NSS (and the financial burden to the society) in Australia is predicted to increase by $35.5 \%-59.3 \%$ in 2021 compared with 2011 and by 1.6- to 4.6-fold in 2051 if current only demographic (first number) or both demographic and incidence trends (second number) continue. Conclusions: Our study demonstrates favorable trends in stroke survivor rates in Australia in the first decade of the new millennium and projects in the foreseeable future significant increases in the absolute numbers of older stroke survivors, especially among those aged 70 years or older and men. Key Words: Stroke survivors-incidence rates-trends-projections-Australia.

(C) 2013 by National Stroke Association
\end{abstract}

\section{Introduction}

Rising life expectancy and declining birth rates have resulted in unprecedented aging of the population worldwide. The continuing demographic transformation, with the oldest age group comprising the fastest growing segment of the population, will further increase the

From the *Department of Geriatric Medicine, The Canberra Hospital, Canberra, Australia; and tAustralian National University Medical School, Canberra, Australia.

Received February 15, 2013; revision received June 4, 2013; accepted June 29, 2013.

Grant support: None.

Address correspondence to Alexander Fisher, MD, PhD, Doc Med Sci, Department of Geriatric Medicine, The Canberra Hospital, PO Box 11, Woden, Canberra, ACT 2606, Australia. E-mail: alex.fisher@ act.gov.au.

$1052-3057 / \$$ - see front matter

(c) 2013 by National Stroke Association

http://dx.doi.org/10.1016/j.jstrokecerebrovasdis.2013.06.035 proportion of seniors in coming years. Increasing age is the strongest risk factor for chronic diseases, including stroke, and dependency. Globally, stroke is projected to be at least until 2030 the second leading cause of mortality ${ }^{1}$ and ranks between the third and the seventh cause of disease burden. ${ }^{2-4}$ Among an estimated 62 million stroke survivors worldwide, ${ }^{4}$ more than half are dependent on others for every day activities ${ }^{5-10}$ and about a third have severe disability or need institutionalization. ${ }^{11}$

In Australia, between 1996-1997 and 2005-2006, the number of hospitalizations for stroke remained stable, and there was an annual reduction of $4.0 \%$ in mortality rate and of $2.2 \%$ in hospitalization rate. ${ }^{12}$ On the other hand, the nonfatal proportion of stroke burden was significantly higher ( $45 \%$ in men and $37 \%$ in women) than previously estimated. ${ }^{13}$

Because the population is aging and stroke mortality is declining faster than stroke incidence, ${ }^{14-18}$ long-term survival after stroke may continue to improve. ${ }^{19}$ In the 
rapidly growing number of very old stroke patients, newer therapies (eg, thrombolysis) may be underused, ${ }^{20,21}$ and the nonfatal burden of stroke-related disability in coming years is likely to rise despite advances in treatment of stroke. However, most epidemiological studies focus mainly on the incidence and mortality rates, whereas age- and sex-specific information regarding stroke survivors is limited and controversial. Little information exists on sex differences and future directions in age-specific incidence rates of stroke survivors, particularly among persons aged 60 years or older, the group in which most stroke events occur and which does not always receive adequate care. $^{22,23}$ Moreover, divergent trends in stroke incidence for men and women were reported. ${ }^{14,24}$ Some researchers found a higher risk of serious cardiovascular events in older male stroke survivors, ${ }^{25}$ whereas others concluded that over coming decades the societal impact of stroke in women will increase as the population ages. ${ }^{26}$

Data on temporal trends and long-term projections of stroke survivors are essential for understanding, planning, and decision making in health-care policies, resource allocation, research, prevention, and treatment of this debilitating disease. Surprisingly, such information is still scarce and controversial.

The objectives of this study were to present (i) ageand sex-specific trends in the incidence rates of stroke survivors 60 years of age and over in an 11-year period from 1999-2000 to 2009-2010 in the Australian Capital Territory (ACT) and (ii) projections of future trends in Australia until 2051.

\section{Methods}

\section{Study Sample}

All strokes occurring in the ACT are treated (hospitalized) only in the Canberra and Calvary public hospitals that make this region an ideal place for populationbased epidemiologic research. We used administrative databases to identify patients discharged with a diagnosis of stroke. We performed a retrospective screening of medical records of all suspected stroke cases in these 2 hospitals. Separations with a principal diagnosis of International Classification of Diseases, 10th Revision (ICD-10-AM) codes 160 through 164 were included in the data set. To prevent double counting, inter- and intrahospital transfers for the same event and readmission were identified by matching date of birth, patient ID number, and admission and separation dates and were excluded from this analysis. Thus, each stroke was counted only once and only if the patient was alive at the time of discharge. Although both patients with first-ever and recurrent stokes were included in the primary database, only the first event was included in the final analysis. Patients younger than 60 years were excluded in the present study. A "stroke survivor" was defined as a person discharged after a nonfatal stroke.

\section{Stroke Classification}

We used the World Health Organization standard definition of stroke. All stroke cases were identified with the first-listed ICD code. Cases were further categorized into subarachnoid hemorrhage (ICD-10-AM 160), intracerebral hemorrhage (ICH, ICD-10-AM 161-162), cerebral infarction (ICD-10-AM 163), and ill-defined (unspecified) stroke (ICD-10-AM 164).

\section{Population Data}

Population estimates were obtained from the Australian Bureau of Statistics (ABS). ${ }^{27}$ The ACT in 2006 (the time of census) had a total population of 334,119 people (1.75\% of 19.9 million Australians), of whom $96.3 \%$ were white, $1.2 \%$ indigenous, and $2.6 \%$ Asians, with $13.8 \%$ aged 60 years and older. Our study covered the period from July 1, 1999, until June 30, 2010. Over this 11-year period, there was a $13.5 \%$ increase in the total ACT population (women $+13.8 \%$ and men $+13.3 \%$ ) with a shift toward higher age: a $50.4 \%$ increase in people aged 60 years or older (women $+48.3 \%$ and men $+53.0 \%$ ) and a $86.8 \%$ increase in people aged 80 years or older (women $+73.5 \%$ and men $+113.1 \%$ ). For Australia, the corresponding proportion figures were $15.5 \%(15.5 \%$, $15.5 \%), 31.7 \%(28.3 \%, 35.7 \%)$, and $52.3 \%$ (42.9\%, 70.0\%). Population estimates from 2011 onward were obtained from the ABS population projection Series B that is based on the 2011 census and trends in fertility rates, life expectancy at birth, and migration and most closely reflects recent trends.

\section{Statistical Analysis}

Annual sex- and age-specific incidence rates (per 100,000 population) were determined using the population data obtained from population census of the ABS. Age- and sex-adjusted stroke survivor rates were estimated using the number of hospital discharges as the numerator and the ACT age and gender population data at mid-year as the denominator. In noncensus years, population estimates from ABS were used. The annual ageand sex-specific incidence rates of stroke survivors were based on the numbers in 5 age groups. Then we calculated incidence rates for 3 age groups: 60-69, 70-79, and 85 years or older. The rates were also determined for stroke subtypes (hemorrhagic, ischemic, and ill defined).

Furthermore, to look into the overall trend in incidence rates of stroke survivors, sex-specific age-standardized incidence rates were calculated by the direct method using the Australian 2006 population aged 60 years or older as the standard. To get age- and sex-adjusted rates, age- and sex-specific rates for each year were multiplied by the corresponding Australian population, which was then divided by the total Australian population of men and women. After standardization, any differences between 
incidence rates of stroke survivors over the study period can be attributed neither to the aging of the population nor to different sex distribution and indicate effects of other factors.

We also estimated in each calendar year changes (\%) in absolute number and sex-specific rates of stroke survivors compared with the previous year and calculated the mean sex-specific annual survivor rate changes (\%) over the 11-year period (1999-2000 to 2009-2010). The secular changes in the incidence rates of stroke survivors over the study period were examined with a Poisson regression model in which time was considered an independent variable, whereas age and sex were covariates. The Poisson regression model used to estimate the number of stroke survivors (NSS) was

$$
\operatorname{NSS}\left(x_{1} ; x_{2}\right)=\mathrm{e}^{a+b_{1} x_{1}+b_{2} x_{2}},
$$

where $x_{1}$ is the age in years and $x_{2}$ the year of stroke occurrence.

The data were analyzed using the Stata software version 10 (StataCorp, College Station, TX). The level of statistical significance was set at 2 -sided $P$ value less than .05 .

\section{Projections}

To project the NSS in 2021 and 2051, we applied ageand sex-specific incidence rates of stroke survivors to the Australian population projections (ABS Series B "medium" data). Two alternative scenarios were assumed in the analysis: (i) incidence rates would remain stable at the average level in the first decade of the 21st century and, thus, predictions are based solely on projected sexand age-specific size of the population at risk and (ii) survival incidence rates would continue to change for the next 40 years at the same rate as during the study period, and therefore, predictions made take into account both factors changing incidence rates and demographic forecast. In this model, the age- and sex-specific incidence rates for 2021 and 2051 were estimated from the Poisson regression equations, and the forecast NSS were calculated multiplying by projected population figures for each age group according to sex. With both models, separate predictions for men and women and for 3 age groups (60-69, 70-79, and 80 years or older) were made. The study was approved by the ACT Health Human Research Ethics Committee.

\section{Results}

\section{General Characteristics of the Stroke Population}

From 1999-2000 to 2009-2010, in the ACT, there were 2228 first-ever stroke hospitalized patients (1111 men and 1117 women) aged 60 years and older, of whom 444 (19.93\%) died (205 [18.5\%] men and 239 [21.4\%] women). From the analysis, we also excluded 150 recurrent stroke events that occurred in 131 elderly patients (2-5 stroke events in each person). The final sample included 1784 discharged alive first-ever stroke patients aged 60 years or older.

Demographic characteristics, stroke type, and absolute NSS during the 11-year period in the ACT and incidence rates according to sex and age are presented in Table 1. Women were significantly older than men when sustaining their stroke (on average 2.7 years, $P<$ .001) and half of them were 80 years or older. Most of the cases were classified as cerebral infarction (47.9\%) and $\mathrm{ICH}(21.1 \%)$. Women more often had a subarachnoid hemorrhage, the least common stroke subtype; no difference in the incidence of other stroke subtypes between genders was observed. The distribution of stroke subtypes did not change over time (data not shown). Taking total number of elderly stroke survivors, men and women were near equal (female to male [F:M] ratio .97); however, among 60- to 69-year-old survivors, $62.3 \%$ were men (F:M ratio .60), whereas in the " 80 years or older" group $55.0 \%$ were woman (F:M ratio 1.22 ). At the end of the study period, in 2010, the age group 80 years or older comprised $4.04 \%$ of the total ACT population (3.20\% among men and $4.85 \%$ among women) and accounted for $44.5 \%$ of stroke survivors (39.4\% among men and $49.8 \%$ among women).

Stroke survival incidence rates (per 100,000 personyears) increased with advancing age in both sexes exponentially and in all 3 age groups were significantly greater in men than in women (F:M ratio .58, .82 , and .70, respectively). The mean standardized rate for men was $20.5 \%$ higher $(P=.0016)$.

\section{Temporal Changes in the Number of Stroke Survivors and Trends in Incidence Rates}

The annual numbers and rates of stroke survivors aged 60 years or older during the 11-year study period are given in Figure 1. The absolute NSS, although varied with a marked drop in 2008, in general, steadily increased among both men and women. In the last 3 years (20072008 to 2009-2010) compared with the first 3 (1999-2000 to 2001-2002), the total NSS increased by $57.5 \%$ (from 369 to 581 ), in men by $73.0 \%$ (from 174 to 301 ), and in women by $43.6 \%$ (from 195 to 280 ). The in-hospital mortality decreased from $37.7 \%$ at the beginning of the study period to $18.7 \%$ at its end, on average an annual 3.8\% decline. During the study period, the temporal sex-specific age-adjusted standardized incidence rates demonstrated a slight insignificant trend toward increasing. The mean annual percentage changes, despite the fluctuations across the study years, showed increases of $4.5 \%(95 \%$ confidence interval [CI]: -7.5 to 16.5$)$ in total, $3.4 \%(95 \%$ CI: -7.5 to 14.3$)$ in men and $7.6 \%(95 \%$ CI: -10.7 to $25.9)$ in women. In the last 3 years compared with the starting 3 years, the incidence rates in total increased on 
Table 1. Demographic characteristics, stroke type, absolute number, and sex-specific age-adjusted standardized* incidence rates of stroke survivors aged $\geq 60$ years in Australian Capital Territory (1999-2000 to 2009-2010)

\begin{tabular}{lccc}
\hline & Women & Men & Total \\
\hline Stroke survivors, n (\%) & 878 & 906 & 1784 \\
Age, y (mean \pm SD) & $78.6 \pm 8.7$ & $75.9 \pm 8.6$ & $77.2 \pm 8.7$ \\
Age groups & $145(16.5)$ & $240(26.5)$ & $385(21.6)$ \\
60-69 y, n (\%) & $296(33.7)$ & $309(34.1)$ & $605(33.9)$ \\
$70-79$ y, n (\%) & $437(49.8)$ & $357(39.4)$ & $794(44.5)$ \\
$\geq 80$ y, n (\%) & & $19(2.1)$ & $65(3.6)$ \\
Types of stroke & $46(5.2)$ & $203(22.4)$ & $377(21.1)$ \\
SAH, n (\%) & $174(19.8)$ & $435(48.0)$ & $854(47.9)$ \\
ICH, n (\%) & $419(47.7)$ & $249(27.5)$ & $488(27.4)$ \\
CI, n $(\%)$ & $239(27.2)$ & & \\
NS, n $(\%)$ & $114(94.0-136.9)$ & $195(168.6-224.4)$ & $154(130.6-180.3)$ \\
Incidence rates per 100,000 person-years $(95 \%$ confidence interval) & $406(367.5-447.5)$ \\
60-69 y & $370(333.2-409.7)$ & $452(411.3-495.7)$ & $1000(939.0-1063.9)$ \\
$70-79$ y & $866(809.3-925.7)$ & $402.1 \pm 50.1$ \\
$\geq 80$ y & $371.3 \pm 62.8$ & $447.5 \pm 56.9$ & \\
Mean $( \pm$ SD) & & & \\
\hline
\end{tabular}

Abbreviations: CI, cerebral infarction; ICH, intracerebral hemorrhage; NS, stroke type not specified; SAH, subarachnoid haemorrhage. *Standardized to 2006 Australian population.

average by $4.5 \%$ (from 375.70 to 392.57 ), but this was confined only to men (on average $+12.9 \%$, from 396.67 to 447.72).

\section{Impact of Age and Time (Year) of Stroke}

The influence of age and calendar stroke year on the number and incidence rates of stroke survivors in the 11-year period was further examined by Poisson regression analysis (Tables 2 and 3). These models explore the combined effects of age (in 5-year intervals) and the calendar period of stroke onset. Generally, the absolute NSS increased with increasing age $\left(b_{1}\right)$ and time of stroke $\left(b_{2}\right)$ by $3.3 \%$ and $5.9 \%$, respectively (Table 2 ). However, stratification for sex revealed that changes related to these factors were different in men and women. The influence of age was larger in women than in men $(5.0 \%$ versus $1.7 \%)$. The effect of calendar year of stroke $\left(b_{2}\right)$, which also significantly and positively influenced the number of survivors, in contrast to age, was more prominent in men than in women $(6.7 \%$ versus $5.1 \%)$. Taken together, among men, the increased NSS during the study period was more influenced by the calendar year of the stroke, than the age, whereas in women both factors have near an equal effect. Of note, the estimates by the Poisson regression model corresponded well with the observed data: in the 11-year period, the calculated and actual NSS for men as for women were practically equal. According to this model, the absolute NSS in the ACT is projected to increase in 2021, compared with 2010, by $93 \%$ (from 224 to 433 ), by $68.5 \%$ in women (from 111 to 187 ), and by $121 \%$ in men (from 113 to 250 ) with further 5.8-,
4.6-, and 7.4-fold increases, respectively, in 2051 (reaching 2530, 855, and 1853 persons).

The incidence rates of stroke survivors in both sexes increased with aging and stroke year (Table 3) and, as would be expected, with a significantly greater influence of age than the time of stroke (in total by $8.8 \%$ and $1.9 \%$, respectively). However, in men, the calendar year of the stroke influenced survival rates to a larger degree than in women ( $2.4 \%$ versus $1.3 \%)$, in whom the influence of age was slightly higher than in men $(9.22 \%$ versus $8.96 \%)$. According to the model for the sex-specific incidence rates (Table 3), explained variances were very high $\left(R^{2} .84-.89\right)$.

\section{Stroke Survivors Forecasts in Australia}

\section{Population changes (2011-2051)}

Over the next 40 years, the Australian population is expected to continue to increase by $7.2 \%$ in 2021 and by $18.7 \%$ in $2051 .^{28}$ The population aged 60 years or older is estimated to increase $34.1 \%(+35.1 \%$ men and $+33.3 \%$ women) by 2021 and $116.9 \% \quad(+119.6 \%$ men and $+114.5 \%$ women) by 2051 . Importantly, the proportion aged 60 years or older in 2021 and 2051 compared with 2011 will increase from $20.7 \%$ to $26.0 \%$ and $38.0 \%$, respectively, including aged those $60-69$ years from $10.5 \%$ to $12.2 \%$ and $15.0 \%$, aged $70-79$ years from $6.2 \%$ to $8.8 \%$ and $12.0 \%$, and aged 80 years or older from $4.1 \%$ to $5.0 \%$ and $11.0 \%$, respectively. Among those aged 60 years or older, the proportion of persons aged 80 years or older is projected to increase from $19.9 \%$ in 2011 to $29.0 \%$ in 2051 and the F:M ratio in this age group will decrease from 1.55 to 1.29 . These age- and sex-specific shifts 

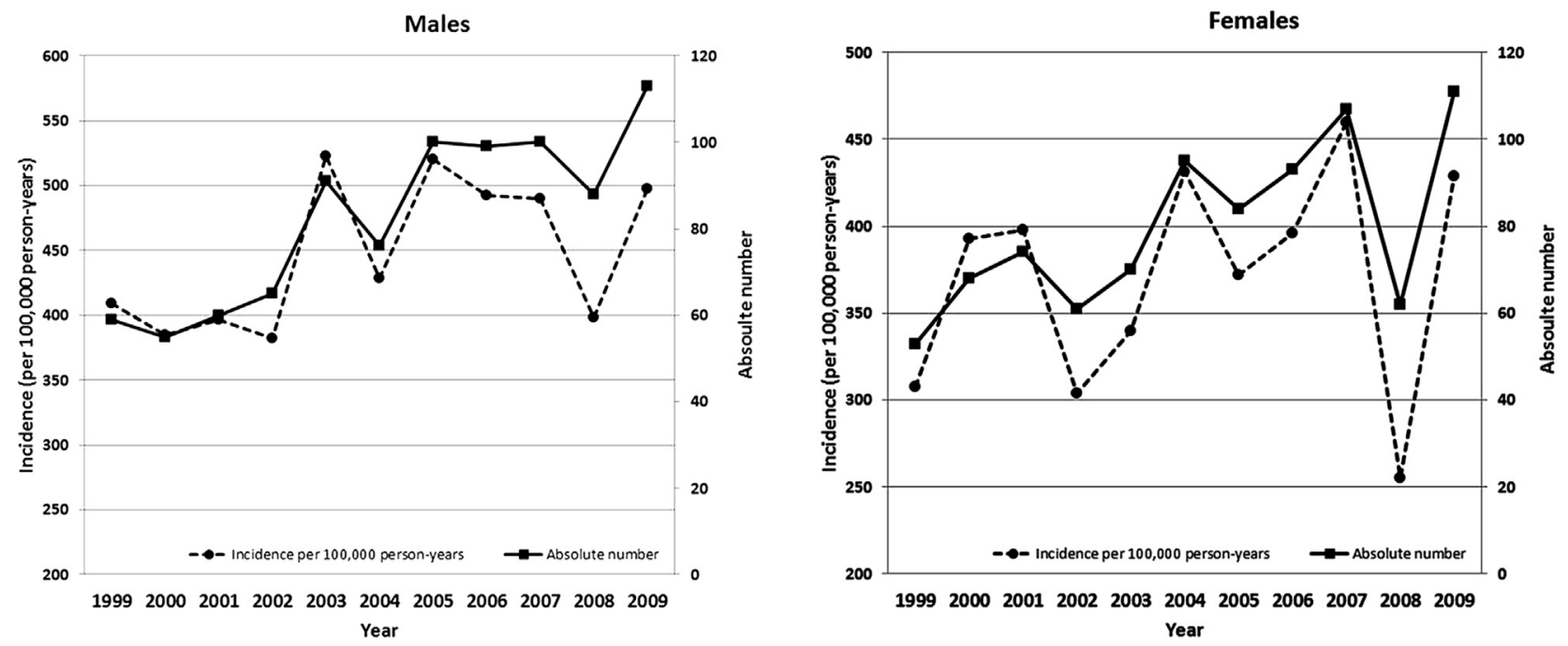

Total

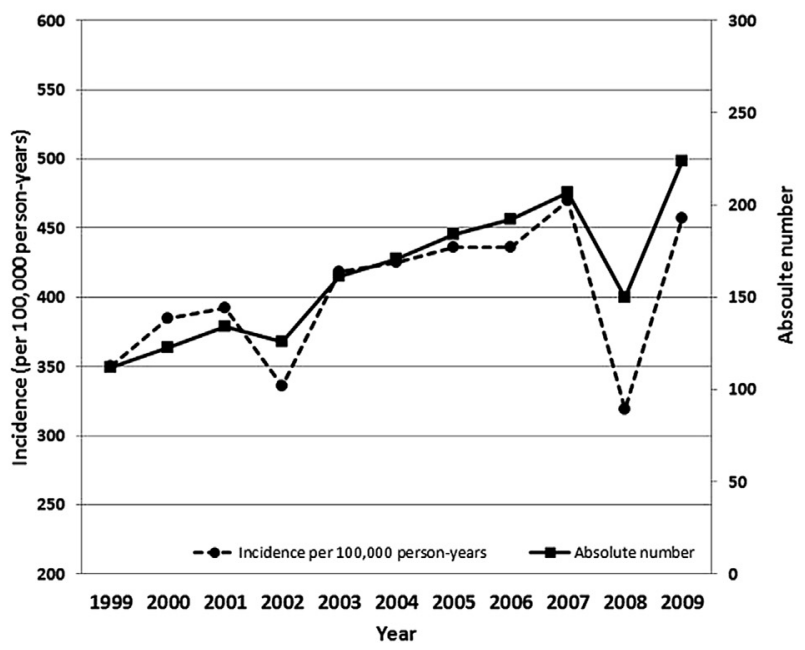

Figure 1. Trends in number (solid lines) and sex-specific age-adjusted standardized (to Australian 2006 population) incidence rates (dashed lines) of stroke survivors in Australian Capital Territory from 1999-2000 to 2009-2010.

toward the most elderly population with an increasing proportion of men are likely to be associated with a higher NSS and more severe disability (as stroke severity worsens significantly with increasing age ${ }^{29,30}$ ).

\section{Stroke survivors in 2021 and 2051}

These data were generated under the assumptions that the incidence rates of stroke survivors for aged 60 years or older in the ACT region are reflective of the national Australian incidence rates of stroke survivors. The numbers of projected stroke survivors for the years 2021 and 2051 according to the 2 models are shown in Table 4 and Figure 2. In model 1, the projections are based only on demographic changes assuming that age- and sex-specific incidence rates of stroke survivors would remain the same over the next 40 years (average for 1999-2000 to 2009-2010 period for each age group). These forecasts suggest that in Australia, compared with 2011, the total NSS aged 60 years or older may increase by $35.5 \%$ in 2021 and by $161.4 \%$ in 2051 , of which $82.0 \%$ in 2021 and $87.3 \%$ in
2051 will be aged 70 years or older, whereas $47.6 \%$ and $60.4 \%$ will be 80 years or older, respectively.

Finally, the gender distribution indicates that the F:M ratio among 60- to 69-year-old and 70- to 79-year-old stroke survivors will not change. However, for the first time among survivors aged 80 years and older, there also will be more men than women: the F:M ratio will decrease from 1.09 in 2011 to .98 in 2021 and to .90 in 2051.

The second model is based on changing of both incidence rates and demography. Assuming age- and sex-specific incidence rates dynamics similar to the 1999-2000 to 2009-2010 period and a population according to the forecast, the expected NSS aged 60 years or older would increase by $59.3 \%$ (by $86.8 \%$ in men and by $43.1 \%$ in women) to the year 2021 and by $436.1 \%$ (by $668.4 \%$ in men and by $384.3 \%$ in women) to 2051 . The F:M ratio is estimated to be .89 in 2021 and .58 in 2051.

Of note, according to both models in 2021, the stroke survivors aged 70-79 years are predicted to have the 
Table 2. Influence of age and calendar year on the number of stroke survivors during the 11-year period (1999-2000 to 2009-2010) in men and women (Poisson regression analysis)

\begin{tabular}{lccc}
\hline & Women & Men & Total \\
\hline Intercept & -102.948 & -132.397 & -117.123 \\
Age $\left(b_{1}\right)$ & .050544 & .01726 & .033224 \\
SE $\left(b_{1}\right)$ & .004185 & .003917 & .002843 \\
$P$ value & $<.001$ & $<.001$ & $<.001$ \\
Year $\left(b_{2}\right)$ & .050719 & .066711 & .058818 \\
SE $\left(b_{2}\right)$ & .010756 & .010648 & .007566 \\
$P$ value & $<.001$ & $<.001$ & $<.001$ \\
$R^{2}$ & .3174 & .1416 & .3181 \\
\hline
\end{tabular}

largest increases $(+54.5 \%$ in men and $+51.4 \%$ in women by model $1 ;+94.6 \%$ and $+67.5 \%$, in men and women, respectively, by model 2), whereas in 2051, the greatest increases will occur in those aged 80 years or older. This is likely because of the fact that the baby-boom generations will reach age more than 70 at year 2021.

Comparisons of the estimates by models 1 and 2 illustrate the contribution from demographic and incidence changes to the projected numbers of stroke survivors and show how it varies between gender and age groups. In 2021, compared with 2011, of the $59.3 \%$ increases in total stroke survivors predicted by model $2,23.8 \%$ may be explained by increasing incidence: $47.4 \%$ in men and $10.4 \%$ in women. Gender difference in the effect of increasing incidence can be observed in the 70- to 79-yearolds $(40.1 \%$ versus $16.1 \%)$ and in those aged 80 years or older $(38.8 \%$ versus $10.7 \%)$, but it is less prominent in the 60 - to 69 -year-olds (35.3\% versus $23.1 \%$ ). Understandably, the gender and age differences in the respective role of incidence changing become significantly higher to the projected numbers of stroke survivors in 2051. The rise in incidence rates will contribute to 2.7 - of 5.4-fold increase in total stroke survivors (4.6- of 7.3-fold in men and 1.3- of 3.8-fold in women), to 2.1- of 3.8-fold in 60to 69 -year-olds (2.8- of 4.5 -fold and 1.3 - of 3.0 -fold in

Table 3. Influence of age and calendar year on the incidence rates of stroke survivors (per 100,000 person-years) during the 11-year period (1999-2000 to 2009-2010) in men and women (Poisson regression analysis)

\begin{tabular}{lccc}
\hline & Women & Men & Total \\
\hline Intercept & -27.033 & -47.6863 & -38.903 \\
Age $\left(b_{1}\right)$ & .092183 & .089552 & .087671 \\
SE $\left(b_{1}\right)$ & .000808 & .000675 & .000739 \\
$P$ value & $<.001$ & $<.001$ & $<.001$ \\
Year $\left(b_{2}\right)$ & .012947 & .023528 & .019125 \\
SE $\left(b_{2}\right)$ & .001827 & .001543 & .001698 \\
$P$ value & $<.001$ & $<.001$ & $<.001$ \\
$R^{2}$ & .8361 & .8774 & .8931 \\
\hline
\end{tabular}

men and women, respectively), to 2.8 - of 5.5-fold in 70to 79-year-olds (3.8- of 6.6-fold and 1.7- of 4.0-fold), and to 1.2- of 4.4-fold in those aged 80 years or older (6.2- of 9.7-fold and 1.0- of 3.9-fold).

\section{Economic Impacts}

One way to evaluate the potential social and economic burden of increasing numbers of stroke survivors in the aging population is to quantify the costs. In Australia, the average costs in the first year were estimated at $\mathrm{AU}$ $\$ 18,956$ (US \$14,361). ${ }^{6,12}$ Applying these data to our projections indicates that expenses for stroke survivors during the first year may reach, according to the first scenario, AU \$445.9 million in 2021 and AU \$860.0 million in 2051 and, according to the second scenario, AU \$693.6 million and AU \$2.34 billion, respectively. Compared with 2011, in the first year after the event, stroke survivors will increase the financial burden by 116.8 million (35.5\%) in 2021 and by 530.9 million $(161.3 \%)$ in 2051 under the circumstances of model 1 and by 258.1 million (59.3\%) in 2021 and 1.9 billion $(436.2 \%)$ in 2051 under the circumstances of model 2.

\section{Discussion}

The main findings of this study are 3-fold: (i) in the ACT in the first decade of the new millennium, the absolute numbers and age-adjusted standardized incidence rates of stroke survivors (measured as a function of age and period) increased among both men and women aged 60 years or older; (ii) the trend toward increased survival rates in both sexes was driven mainly by population aging, whereas the effect of year of stroke was more pronounced in men compared with women; and (iii) the absolute NSS in Australia is predicted to increase by $35.5 \%-59.3 \%$ in 2021 compared with 2011 and by 1.6- to 4.6-fold in 2051 if current demographic (first number) or both demographic and incidence trends (second number) continue.

\section{Stroke Survivor Trends in the First Decade of the 21st Century}

The increase in elderly stroke survivors could be because of higher stroke incidence, declines in mortality, or both. Our observation of significantly decreasing inhospital mortality (3.8\% per year) is similar to the $4.0 \%$ annual reduction in stroke mortality rate in Australia between 1996-1997 and 2005-2006 ${ }^{12}$ and consistent with other studies. In past decades, data from high-income countries, , $^{3,15,16,31-38}$ including Australia ${ }^{12,39-42}$ and New Zealand, ${ }^{14}$ indicated that stroke incidence rates, case fatality, and mortality rates had fallen (for whites but not for blacks and other ethnic groups). However, other studies covering the last 2 decades in the same countries reported stable or even rising stroke incidence. ${ }^{17,19,43-49}$ 
Table 4. Forecast number of stroke survivors aged $\geq 60$ years in Australia to 2021 and 2051 according to 2 models

\begin{tabular}{|c|c|c|c|c|c|c|c|c|c|c|c|c|}
\hline \multirow[b]{2}{*}{ Age group } & \multicolumn{3}{|c|}{ Women } & \multicolumn{3}{|c|}{ Men } & \multicolumn{3}{|c|}{ Total } & \multicolumn{3}{|c|}{$\mathrm{F}: \mathrm{M}$ ratio } \\
\hline & 2011 & 2021 & 2051 & 2011 & 2021 & 2051 & 2011 & 2021 & 2051 & 2011 & 2021 & 2051 \\
\hline \multicolumn{13}{|c|}{ Model 1 (incidence rates unchanged, similar to the average in the $1999-2000$ to $2009-2010$ period) } \\
\hline $60-69$ & 1265 & 1604 & 2136 & 2132 & 2630 & 3635 & 3392 & 4244 & 5756 & .59 & .61 & .59 \\
\hline $70-79$ & 2509 & 3798 & 5726 & 2791 & 4313 & 6515 & 5286 & 8081 & 12,196 & .9 & .88 & .88 \\
\hline$\geq 80$ & 4571 & 5649 & 13,370 & 4199 & 5769 & 14,783 & 8681 & 11,198 & 27,418 & 1.09 & .98 & .90 \\
\hline Total & 8345 & 11,051 & 21,232 & 9122 & 12,712 & 24,933 & 17,359 & 23,523 & 45,370 & .91 & .87 & .85 \\
\hline \multicolumn{13}{|c|}{ Model 2 (incidence rates changed as during the $1999-2000$ to $2009-2010$ period) } \\
\hline $60-69$ & 1559 & 2337 & 4716 & 2531 & 4016 & 11,460 & 4109 & 6390 & 15,743 & 62 & .58 & .41 \\
\hline $70-79$ & 2423 & 4058 & 9727 & 3485 & 6783 & 21,440 & 5824 & 10,606 & 29,964 & .70 & .60 & .45 \\
\hline$\geq 80$ & 7883 & 10,584 & 31,152 & 4718 & 8313 & 45,731 & 13,044 & 19,595 & 77,476 & 1.67 & 1.27 & .68 \\
\hline Total & 11,864 & 16,979 & 45,595 & 10,735 & 19,113 & 78,631 & 22,977 & 36,591 & 123,182 & 1.16 & .89 & .58 \\
\hline
\end{tabular}

The absolute number of stroke events and prevalence did not decrease $e^{44,50}$ and even continue to rise. ${ }^{17,41}$ At the end of the past century, in several studies, the declining stroke mortality could not be explained by changes in stroke incidence. ${ }^{51-53}$ Diverging trends in stroke mortality and incidence have been recently reported in the United States $^{54}$ and some European countries. ${ }^{45}$ In Australia up to 2005-2006, the declines in deaths from stroke were about 2 times higher than reduction in hospitalizations (4.0\% versus $2.2 \%) .{ }^{12}$ It appears, therefore, that declining mortality rates as a result of improvements in prevention, acute stroke management, and therapy, 35,56 may had a significant effect on the NSS. The plausible and encouraging decline in stroke deaths occurred in parallel with demographic changes. Given that the elderly population who are those most at risk constitute the fastest growing segment of the population, it is not surprising that over the last 11-year period in the ACT, concomitant with declining stroke mortality, the absolute number and incidence rates of stroke survivors aged 60 years or older increased.

Some prior studies reported trends in stroke incidence only by period. Our study illustrates the advantage and importance when analyzing secular trends to use models that account age and time (calendar year) of stroke simultaneously. The age effect reflects mainly biological factors, whereas the period effect includes the influence of preventive and treatment procedures. This approach revealed that, in the ACT, the incidence rates of stroke survivors in the first decade of the 21st century were increasing in men and women positively influenced by both age and year of the stroke, but in men, the effect of time was more pronounced. The increase of stroke rates with age is a well-known phenomenon. ${ }^{57}$ In our study, among stroke survivors, $49.8 \%$ of women and $39.4 \%$ of men were 80 years of age and older. The causes of sex differences in regard to the stroke year are less clear and likely to be multifactorial, indicating possible more effective control of cardiovascular risk factors and/or more intensive care management in the acute phase of the stroke in men compared with women ${ }^{24,58-60}$ who are older and have greater comorbidity. ${ }^{61}$

Worldwide, stroke incidence and mortality rates are higher in men than in women, but more women are affected (because of greater longevity) and possibly are more severely ill with greater disability and dependency. ${ }^{11,57,58,61-67}$ Some studies report divergent trends in stroke incidence for men and women ${ }^{14,24}$ and a higher risk of serious cardiovascular events in older male stroke survivors. $^{25}$

With respect to the F:M ratio for the absolute NSS, our data in line with other reports ${ }^{68}$ show a steep rise from .60 in the 60- to 69-year old to 1.22 among those aged 80 years or older. The F:M ratio for incidence rates also increases with aging, but the highest level occurs in the 70- to 79 -year-old group $(.58, .81$, and .70 in the 3 age groups, respectively). The findings of higher stroke survivor rates among men compared with women aged 60-80 years in this study are consistent with several population-based studies of stroke incidence. $8,11,66,69,70$

\section{Future Trends and Implications}

Future trends in absolute numbers of older stroke survivors are determined by population growth and aging, stroke incidence, and stroke mortality rates.

Because the observed variability in temporal incidence rates was a matter of concern in long-term projections, we concentrated on 2 models reflecting stable and increasing incidence rates of stroke survivors. Our data indicate a very significant increase in the future NSS in Australia and consequently a shift from a mortality burden toward a morbidity burden similar to that recently predicted for Western Europe. ${ }^{15}$

In Australia, the forecast based only on population growth and age-related changes indicates a 35.5\% (39.4\% in men and $32.4 \%$ in women) increase in stroke survivors in 2021 and $161.4 \%$ (154.4\% and 173.3\% in men and women, respectively) in 2051 compared with 2011. Importantly, more than $80 \%$ of stroke survivors 

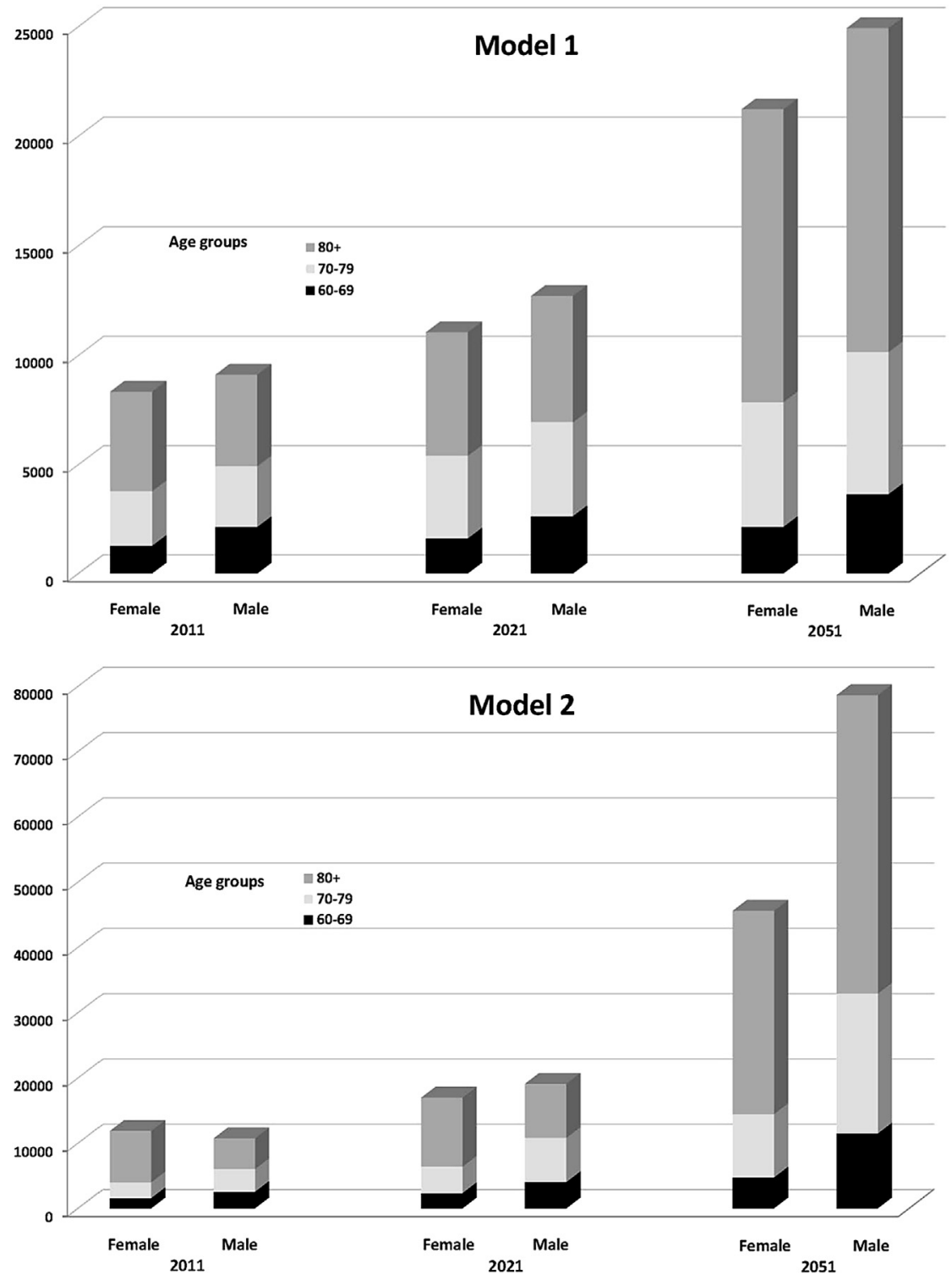

Figure 2. Forecast number of stroke survivors aged 60 years and older in Australia to 2021 and 2051 according to 2 models. In model 1 , the projections are based on demographic changes assuming that age- and sex-specific incidence rates of stroke survivors would remain unchanged similar to the average in the 1999-2000 to 2009-2010 period. In model 2, the projections are based on changing of both incidence rates (as during the 1999-2000 to 2009-2010 period) and demography. will be 70 years or older and about a half 80 years or older with a prevalence of men in all age groups. In Europe, solely because of the demographic changes, the annual number of strokes is predicted to increase from 1.1 million in 2000 to 1.5 million $(+36.4 \%)$ in $2025 .{ }^{71}$ It is expected that the negative effect of stroke on life expectancy in 7 western European countries would strongly decline: in 2030, about .5 years in men and less than 1 year in women. ${ }^{15}$ Data presented in our conservative model 1 are comparable with estimates from Europe. Of note, in Australia, the number of surviving poststroke individuals tripled from 1996 to $2010 .^{72}$

According to the second model, which includes increases in incidence rates in addition to demographic changes, the projected number of older stroke survivors may increase by $59.3 \%$ in 2021 and by $436.1 \%$ in 2051 . For men aged 70 years or older, the effect of increasing rates on the total number of projected survivors in 2021 is 2.5 times (for 70- to 79-year-olds) to 3.8 times (for those aged 80 years or older) higher than in women; in 2051, the corresponding figures are 2.2 and 6.0 times. Both models suggest that the societal impact of stroke in older men will continue to increase (in all age groups, F:M ratio is less then 1.0, except for those aged 80 years or older in 2021 in model 2) indicating that it is necessary to pay more attention to stroke prevention in the elderly men, even though the stroke mortality is higher in women.

Taken together, these forecasts suggest that over the next few decades a very significant increase in numbers of older stroke survivors is to be anticipated. In 2021, the age group 80 years or older will comprise $5.0 \%$ of the population and account for $47.6 \%$ (model 1 ) to $53.6 \%$ (model 2 ) of stroke survivors, and in 2051, the corresponding figures will be $11.0 \%$ and $60.4 \%-62.9 \%$. These significant increases 
in age-specific prevalence of stroke survivors will lead to increases in the demand for health care and costs, including the need of rehabilitation and long-term care beds. The association between older age and a poorer short- and long-term poststroke prognosis is well documented. ${ }^{40,73,74}$ More disabled stroke survivors and a very substantial economic burden might be expected as long-term costs account for about $80 \%$ of total stroke costs, ${ }^{75,76}$ although in the first year poststroke $30 \%-50 \%$ of the costs fall on acute hospitalization. ${ }^{76,77}$

It was estimated that in Australia, the cost of treating 1 stroke patient in the first year after the event is approximately AU $\$ 18,956$ (US $\$ 14,361),{ }^{78}$ the lifetime costs per first-ever case for ischemic stroke is AU $\$ 57,106$ and for ICH stroke AU $\$ 49,995,{ }^{79}$ and the total health-care expenditure on stroke in 2004-2005 was AU \$546 million. ${ }^{72}$ In Sweden, the average lifetime cost per individual is $€ 84,500^{77}$ and in United States US $\$ 103,576 .{ }^{80}$ Despite variations in the published costs of stroke between countries, data from Australia and other developed countries are comparable. In Australia, New Zealand, and Canada, the mean total cost (per case), during the first year after a first-ever stroke, was estimated at US $\$ 16,762^{81}$ and in 30 European countries (on average) $€ 21,000 .^{82}$ Based on the mean stroke costs in Australia during the first year after the event, we estimated that expenses from increase of new stroke victims would increase the financial burden to the society by $35.5 \%$ (model 1) to $59.3 \%$ (model 2) in 2021 and by $161.3 \%-436.2 \%$ in 2051 compared with 2011.

In coming decades, stroke burden will continue to shift away from premature death to years lived with disability. ${ }^{83,84}$ Stroke survivors care may constitute a significant economic challenge for the health-care system in Australia and worldwide. The NSS is expected to rise globally from 62 million in 2005 to 67 million in 2015 and 77 million in 2030, ${ }^{4}$ and on average, the duration of survival after first-ever stroke is estimated to be 8.2 (women) to 9.0 (men) years. ${ }^{18}$ Therefore, prevention of stroke-related disability and improving survivorship care may become more important. ${ }^{15,54,84}$ More research is needed to better understand the projected burden of the disease on patients, families, the health-care system, and society as a whole and properly address the issue of needs and costs when resources are scarce. The goals are to develop new preventive and treatment approaches that will significantly decrease the number of stroke victims and to improve the acute and long-term poststroke care that may markedly reduce the number of disabled persons. A co-ordinated plan of action of policy makers, health providers, and researchers to address the challenges of this debilitating disease with long-term consequences is warranted.

\section{Strength and Limitations}

The present analysis was undertaken on data collected continuously over an 11-year period and included all patients hospitalized with a stroke in a well-defined region. Corrections for recurrent strokes, double counting, standardization, and evaluation of incidence trends by models that combine patients' age and period of the event are other strengths of the study. Several limitations to our study should be considered. First, there is some potential for missing cases, especially of out-of-hospital strokes and patients who died at home. However, the number of nonhospitalized stroke survivors should be minimal as in ACT any patient with symptomatic stroke can be admitted only to 1 of 2 public hospitals, the stroke admittance rate in ACT (as in other Australian state ${ }^{12,39,41}$ ) is high, and ACT has a well-established full-time medical emergency and transport system. Second, we have not evaluated the incidence trends of stroke survivors in regard to stroke subtypes and stroke severity because of small numbers. Third, the study was conducted in an area with a predominantly white population, limiting the generalizability of our findings. Fourth, we used observed trends in incidence rates of stroke survivors in 1999-2000 to 2009-2010 in the ACT as the basis to make projections for the future. Although this is a reasonable approximation, data may be not sufficient to predict the future NSS accurately. Clearly, the main limitation to such extrapolation-based projections is that they cannot take into account unforeseen changes either in future incidence and mortality rates nor in demographics and in a variety of confounding social and political events. Socioeconomic and ethnic differences between Australian states and territories also add uncertainty to the predictions. Thus, our forecasts, which are based on currently available data and reflect the implications of recently observed trends on future health trajectories and disease burden, may provide a realistic long-term perspective but should be interpreted cautiously.

\section{Conclusions}

Our study demonstrates favorable trends in stroke survivor rates of older ( $\geq 60$ years) patients in Australia in the first decade of the new millennium and projects in the foreseeable future significant increases in the absolute numbers of stroke survivors, especially among those aged 70 years or older and men. There is an urgent need to improve stroke prevention and treatment efforts to decrease the projected social and economic disease burden.

\section{References}

1. Mathers CD, Loncar D. Projections of global mortality and burden of disease from 2002 to 2030. PLoS Med 2006;3:e442.

2. Begg SJ, Vos T, Barker B, et al. Burden of disease and injury in Australia in the new millennium: measuring health loss from diseases, injuries and risk factors. Med J Aust 2008;188:36-40. 
3. Ovbiagele B, Nguyen-Huynh MN. Stroke epidemiology: advancing our understanding of disease mechanism and therapy. Neurotherapeutics 2011;8:319-329.

4. Strong K, Mathers C, Bonita R. Preventing stroke: saving lives around the world. Lancet Neurol 2007;6:182-187.

5. Adamson J, Beswick A, Ebrahim S. Is stroke the most common cause of disability? J Stroke Cerebrovasc Dis 2004;13:171-177.

6. Hankey GJ, Jamrozik K, Broadhurst RJ, et al. Long-term disability after first-ever stroke and related prognostic factors in the Perth Community Stroke Study, 19891990. Stroke 2002;33:1034-1040.

7. Hardie K, Hankey GJ, Jamrozik K, et al. Ten-year risk of first recurrent stroke and disability after first-ever stroke in the Perth Community Stroke Study. Stroke 2004; 3:731-735.

8. Rothwell PM, Coull AJ, Giles MF, et al. Change in stroke incidence, mortality, case-fatality, severity, and risk factors in Oxfordshire, UK from 1981 to 2004 (Oxford Vascular Study). Lancet 2004;363:1925-1933.

9. Sturm JW, Dewey HM, Donnan GA, et al. Handicap after stroke: how does it relate to disability, perception of recovery, and stroke subtype?: The North East Melbourne Stroke Incidence Study (NEMESIS). Stroke 2002; 33:762-768

10. Wolfe CD, Smeeton NC, Coshall C, et al. Survival differences after stroke in a multiethnic population: follow-up study with the South London Stroke Register. BMJ 2005; 331:431.

11. Petrea RE, Beiser AS, Seshadri S, et al. Gender differences in stroke incidence and poststroke disability in the Framingham Heart Study. Stroke 2009;4:1032-1037.

12. Thrift AG, Tong B, Senes $S$, et al. No evidence for an epidemic of stroke with the ageing of the population. Neuroepidemiology 2012;38:268-273.

13. Katzenellenbogen JM, Begg S, Somerford P, et al. Disability burden due to stroke in Western Australia: new insights from linked data sources. Int J Stroke 2010; 5:269-277.

14. Anderson CS, Carter KN, Hackett ML, et al. Trends in stroke incidence in Auckland, New Zealand, during 1981 to 2003. Stroke 2005;36:2087-2093.

15. Kunst AE, Amiri M, Janssen F. The decline in stroke mortality: exploration of future trends in 7 Western European countries. Stroke 2011;42:2126-2130.

16. Roger VL, Go AS, Lloyd-Jones DM, et al. Executive summary: heart disease and stroke statistics-2012 update: a report from the American Heart Association. Circulation 2012;125:188-197.

17. Sivenius J, Torppa J, Tuomilehto J, et al. Modelling the burden of stroke in Finland until 2030. Int J Stroke 2009; 4:340-345.

18. Tobias M, Cheung J, Carter K, et al. Stroke surveillance: population-based estimates and projections for New Zealand. Aust N Z J Public Health 2007;31:520-525.

19. Hallstrom B, Jonsson AC, Nerbrand C, et al. Stroke incidence and survival in the beginning of the 21st century in southern Sweden: comparisons with the late 20th century and projections into the future. Stroke 2008;39:10-15.

20. Foerch C, Sitzer M, Steinmetz H, et al. Future demographic trends decrease the proportion of ischemic stroke patients receiving thrombolytic therapy: a call to set-up therapeutic studies in the very old. Stroke 2009; 40:1900-1902.

21. Singer OC, Hamann GF, Misselwitz B, et al. Time trends in systemic thrombolysis in a large hospital-based stroke registry. Cerebrovasc Dis 2012;33:316-321.
22. Bhalla A, Grieve R, Tilling K, et al. Older stroke patients in Europe: stroke care and determinants of outcome. Age Ageing 2004;33:618-624.

23. Kee YY, Brooks W, Bhalla A. Do older patients receive adequate stroke care? An experience of a neurovascular clinic. Postgrad Med J 2009;85:115-118.

24. Wieberdink RG, Ikram MA, Hofman A, et al. Trends in stroke incidence rates and stroke risk factors in Rotterdam, the Netherlands from 1990 to 2008. Eur J Epidemiol 2012;27:287-295.

25. Towfighi A, Markovic D, Ovbiagele B. Temporal trends in risk of future cardiac events among stroke survivors in the United States. Int J Stroke 2012;7:207-212.

26. Reeves MJ, Lisabeth LD. The confounding issue of sex and stroke. Neurology 2010;74:947-948.

27. Australian Bureau of Statistics. 3101.0-Australian Demographic Statistics; 2012. Available at: http://www.abs. gov.au/ausstats/abs@.nsf/mf/3101.0. Accessed December, 2012.

28. Australian Bureau of Statistics. 1370.0.55.001-Measures of Australia's Progress: Summary Indicators; 2012. Available at: http://www.abs.gov.au/ausstats/abs@.nsf/mf/ 1370.0.55.001. Accessed December, 2012.

29. Andersen KK, Olsen TS. One-month to 10-year survival in the Copenhagen stroke study: interactions between stroke severity and other prognostic indicators. J Stroke Cerebrovasc Dis 2011;20:117-123.

30. Feigin VL, Barker-Collo S, Parag V, et al. Auckland Stroke Outcomes Study. Part 1: gender, stroke types, ethnicity, and functional outcomes 5 years poststroke. Neurology 2010;75:1597-1607.

31. Bejot Y, Rouaud O, Durier J, et al. Decrease in the stroke case fatality rates in a French population-based twentyyear study. A comparison between men and women. Cerebrovasc Dis 2007;24:439-444.

32. Carandang R, Seshadri S, Beiser A, et al. Trends in incidence, lifetime risk, severity, and 30-day mortality of stroke over the past 50 years. JAMA 2006;296:2939-2946.

33. Fang J, Alderman MH, Keenan NL, et al. Declining US stroke hospitalization since 1997: National Hospital Discharge Survey, 1988-2004. Neuroepidemiology 2007; 29:243-249.

34. Feigin VL, Lawes CM, Bennett DA, et al. Worldwide stroke incidence and early case fatality reported in 56 population-based studies: a systematic review. Lancet Neurol 2009;8:355-369.

35. Kleindorfer DO, Khoury J, Moomaw CJ, et al. Stroke incidence is decreasing in whites but not in blacks: a population-based estimate of temporal trends in stroke incidence from the Greater Cincinnati/Northern Kentucky Stroke Study. Stroke 2010;41:1326-1331.

36. Meretoja A, Kaste M, Roine RO, et al. Trends in treatment and outcome of stroke patients in Finland from 1999 to 2007. PERFECT Stroke, a nationwide register study. Ann Med 2011;43(suppl 1):S22-S30.

37. Towfighi A, Saver JL. Stroke declines from third to fourth leading cause of death in the United States: historical perspective and challenges ahead. Stroke 2011; 42:2351-2355.

38. Webster F, Saposnik G, Kapral MK, et al. Organized outpatient care: stroke prevention clinic referrals are associated with reduced mortality after transient ischemic attack and ischemic stroke. Stroke 2011;42:3176-3182.

39. Islam MS, Anderson CS, Hankey GJ, et al. Trends in incidence and outcome of stroke in Perth, Western Australia during 1989 to 2001: the Perth Community Stroke Study. Stroke 2008;39:776-782. 
40. Jamrozik K, Broadhurst RJ, Lai N, et al. Trends in the incidence, severity, and short-term outcome of stroke in perth, Western Australia. Stroke 1999;30:2105-2111.

41. Marsden DL, Spratt NJ, Walker R, et al. Trends in stroke attack rates and case fatality in the Hunter region, Australia 1996-2008. Cerebrovasc Dis 2010;30:500-507.

42. Senes S. How we manage stroke in Australia. AIHW cat. no. CVD 31. Canberra, Australia: Australian Institute of Health and Welfare, 2006.

43. Appelros P, Nydevik I, Seiger A, et al. High incidence rates of stroke in Orebro, Sweden: further support for regional incidence differences within Scandinavia. Cerebrovasc Dis 2002;14:161-168.

44. Bejot $Y$, Touze E, Jacquin A, et al. Epidemiology of stroke. Med Sci (Paris) 2009;25:727-732.

45. Harmsen P, Wilhelmsen L, Jacobsson A. Stroke incidence and mortality rates 1987 to 2006 related to secular trends of cardiovascular risk factors in Gothenburg, Sweden. Stroke 2009;40:2691-2697.

46. Khellaf M, Quantin C, d'Athis P, et al. Age-period-cohort analysis of stroke incidence in Dijon from 1985 to 2005. Stroke 2010;41:2762-2767.

47. Sugama C, Isa K, Okumura K, et al. Trends in the Incidence of Stroke and Cardiovascular Risk Factors on the Isolated Island of Okinawa: The Miyakojima Study. J Stroke Cerebrovasc Dis 2012 (in press).

48. Terent A. Trends in stroke incidence and 10-year survival in Soderhamn, Sweden, 1975-2001. Stroke 2003;34:1353-1388.

49. Thorvaldsen P, Kuulasmaa K, Rajakangas AM, et al. Stroke trends in the WHO MONICA project. Stroke 1997;28:500-506.

50. Lovelock CE, Molyneux AJ, Rothwell PM. Change in incidence and aetiology of intracerebral haemorrhage in Oxfordshire, UK, between 1981 and 2006: a populationbased study. Lancet Neurol 2007;6:487-493.

51. Harmsen P, Tsipogianni A, Wilhelmsen L. Stroke incidence rates were unchanged, while fatality rates declined, during 1971-1987 in Goteborg, Sweden. Stroke 1992;23:1410-1415.

52. Jorgensen HS, Plesner AM, Hubbe P, et al. Marked increase of stroke incidence in men between 1972 and 1990 in Frederiksberg, Denmark. Stroke 1992;23:1701-1704.

53. McGovern PG, Burke GL, Sprafka JM, et al. Trends in mortality, morbidity, and risk factor levels for stroke from 1960 through 1990. The Minnesota Heart Survey. JAMA 1992;268:753-759.

54. Burke JF, Lisabeth LD, Brown DL, et al. Determining stroke's rank as a cause of death using multicause mortality data. Stroke 2012;43:2207-2211.

55. Asplund K. What MONICA told us about stroke. Lancet Neurol 2005;4:64-68.

56. O'Donnell MJ, Xavier D, Liu L, et al. Risk factors for ischaemic and intracerebral haemorrhagic stroke in 22 countries (the INTERSTROKE study): a case-control study. Lancet 2010;376:112-123.

57. Reeves MJ, Bushnell CD, Howard G, et al. Sex differences in stroke: epidemiology, clinical presentation, medical care, and outcomes. Lancet Neurol 2008;7:915-926.

58. Gargano JW, Wehner S, Reeves M. Sex differences in acute stroke care in a statewide stroke registry. Stroke 2008;39:24-29.

59. Reid JM, Dai D, Gubitz GJ, et al. Gender differences in stroke examined in a 10-year cohort of patients admitted to a Canadian teaching hospital. Stroke 2008; 39:1090-1095.

60. Towfighi A, Ovbiagele B, Saver JL. Therapeutic milestone: stroke declines from the second to the third leading organ- and disease-specific cause of death in the United States. Stroke 2010;41:499-503.

61. Gall SL, Donnan G, Dewey HM, et al. Sex differences in presentation, severity, and management of stroke in a population-based study. Neurology 2010;74:975-981.

62. Appelros P, Stegmayr B, Terent A. A review on sex differences in stroke treatment and outcome. Acta Neurol Scand 2010;121:359-369.

63. Bushnell CD, Hurn P, Colton C, et al. Advancing the study of stroke in women: summary and recommendations for future research from an NINDS-Sponsored Multidisciplinary Working Group. Stroke 2006;37: 2387-2399.

64. Kapral MK, Degani N, Hall R, et al. Gender differences in stroke care and outcomes in Ontario. Womens Health Issues 2011;21:171-176.

65. Lai SM, Duncan PW, Dew P, et al. Sex differences in stroke recovery. Prev Chronic Dis 2005;2:A13.

66. Sealy-Jefferson S, Wing JJ, Sanchez BN, et al. Age- and ethnic-specific sex differences in stroke risk. Gend Med 2012;9:121-128.

67. Towfighi A, Markovic D, Ovbiagele B. Persistent sex disparity in midlife stroke prevalence in the United States. Cerebrovasc Dis 2011;31:322-328.

68. Lofmark U, Hammarstrom A. Evidence for agedependent education-related differences in men and women with first-ever stroke. Results from a community-based incidence study in northern Sweden. Neuroepidemiology 2007;28:135-141.

69. Kissela BM, Khoury JC, Alwell K, et al. Age at stroke: temporal trends in stroke incidence in a large, biracial population. Neurology 2012;79:1781-1787.

70. Towfighi A, Markovic D, Ovbiagele B. Recent patterns of sex-specific midlife stroke hospitalization rates in the United States. Stroke 2011;42:3029-3033.

71. Truelsen T, Piechowski-Jozwiak B, Bonita R, et al. Stroke incidence and prevalence in Europe: a review of available data. Eur J Neurol 2006;13:581-598.

72. AIHW. Health expenditure Australia 2008-09. Health and welfare expenditure series no. 42. Cat. no. HWE 51. Available at: http://www.aihw.gov.au/publication-detail/? id $=6442472450 ; 2010$. Accessed December, 2012.

73. Kammersgaard LP, Jorgensen HS, Reith J, et al. Short- and long-term prognosis for very old stroke patients. The Copenhagen Stroke Study. Age Ageing 2004;33:149-154.

74. Sharma JC, Fletcher S, Vassallo M. Strokes in the elderly-higher acute and 3-month mortality-an explanation. Cerebrovasc Dis 1999;9:2-9.

75. Caro JJ, Huybrechts KF. Stroke treatment economic model (STEM): predicting long-term costs from functional status. Stroke 1999;30:2574-2579.

76. Fattore G, Torbica A, Susi A, et al. The social and economic burden of stroke survivors in Italy: a prospective, incidence-based, multi-centre cost of illness study. BMC Neurol 2012;12:137.

77. Persson J, Ferraz-Nunes J, Karlberg I. Economic burden of stroke in a large county in Sweden. BMC Health Serv Res 2012;12:341.

78. Dewey HM, Thrift AG, Mihalopoulos C, et al. Cost of stroke in Australia from a societal perspective: results from the North East Melbourne Stroke Incidence Study (NEMESIS). Stroke 2001;32:2409-2416.

79. Cadilhac DA, Carter R, Thrift AG, et al. Estimating the long-term costs of ischemic and hemorrhagic stroke for Australia: new evidence derived from the North East Melbourne Stroke Incidence Study (NEMESIS). Stroke 2009;40:915-921. 
80. Taylor TN, Davis PH, Torner JC, et al. Lifetime cost of stroke in the United States. Stroke 1996;27:1459-1466.

81. Luengo-Fernandez R, Gray AM, Rothwell PM. Costs of stroke using patient-level data: a critical review of the literature. Stroke 2009;40:e18-e23.

82. Olsen TS, Andersen ZJ, Andersen KK. Age trajectories of stroke case fatality: leveling off at the highest ages. Epidemiology 2011;22:432-436.
83. Cadilhac DA, Dewey HM, Vos T, et al. The health loss from ischemic stroke and intracerebral hemorrhage: evidence from the North East Melbourne Stroke Incidence Study (NEMESIS). Health Qual Life Outcomes 2010;8:49.

84. Wolfe CD, Crichton SL, Heuschmann PU, et al. Estimates of outcomes up to ten years after stroke: analysis from the prospective South London Stroke Register. PLoS Med 2011;8:e1001033. 